\title{
Les municipalités de centralité, pôles de développement en milieu rural : une tentative de définition
}

\author{
Peggy Bachman et Jean Langevin ${ }^{1}$ \\ Union des municipalités du Québec
}

\section{Introduction}

Le présent article a pour objectif de présenter une définition par caractérisation qualitative des municipalités urbaines en milieu rural québécois. Ces municipalités ont été désignées sous le vocable de municipalités de centralité. Il s'agit des municipalités qui ne se retrouvent pas à l'intérieur des périmètres d'une agglomération de recensement (AR) ou d'une région métropolitaine de recensement (RMR), au sens statistique, mais qui offrent certaines caractéristiques comparables.

Le développement du territoire des régions du Québec a connu plusieurs formes d'intervention au cours des dernières décennies. La tendance vers un développement socio-économique dicté par des politiques pensées et contrôlées par le gouvernement du Québec a connu un apogée pendant les années 19701980. À la suite d'un Le développement du territoire des régions du Québec a connu plusieurs formes d'intervention au cours des dernières décennies.

constat sur le manque de

souplesse de ces politiques quant aux disparités des régions entre elles et du peu d'intérêt qu'elles ont suscité pour l'initiative et la prise en charge par le milieu, les acteurs du développement régional ont révisé leurs orientations pour favoriser désormais la mise en place de structures décentralisées afin de permettre un essor propre à chacune des régions du Québec.

C'est dans cet esprit que les Municipalités régionales de comté (MRC) et les Conseils régionaux de développement (CRD) ont été créés.

Dans son objectif d'accroître la décentralisation des pouvoirs de développement régional, le gouvernement amorçait en 1995 une autre vague de réorgani- sations municipales. C'est effectivement en 1995 que le ministère des Affaires municipales et de la Métropole lançait sa politique de consolidation des communautés locales. C'est dans le contexte de cette réorganisation que l'idée de la municipalité de centralité a germé.

\section{L'émergence d'un réseau de municipalités en milieu rural}

L'émergence d'un réseau de municipalités en milieu rural, dites de centralité, provient principalement de l'initiative gouvernementale de consolider les communautés locales. Par cette initiative, le gouvernement voulait mettre un terme au trop grand fractionnement de la carte municipale québécoise. Cette situation, disait-on, était responsable d'une concurrence stérile entre les municipalités, d'une faiblesse d'organisation dans l'aménagement $\mathrm{du}$ territoire et de grandes lacunes dans la capacité financière de développement socio-économique de celles-ci. La politique ministérielle allait donc engendrer un fort courant de regroupements municipaux à travers le Québec entier. Ce mouvement de consolidations des structures municipales à grande échelle a conduit à créer une nouvelle typologie des municipalités québécoises. Ainsi, la carte municipale québécoise devenait désormais clairement divisée non seulement en territoires régionaux, comme celui des MRC, mais de surcroît, les municipalités locales entre elles se retrouvaient dans différentes catégories. Ce départage entre catégories a été fondé sur des références strictement de statistiques urbaines d'agglomérations de recensement et de régions métropolitaines de recensement. De la sorte, mises à part les municipalités qui répondaient à ces critères statistiques de rassemblement, toutes les autres, ce qui 
représente un nombre considérable, se voyaient réunies, confondues et classifiées comme appartenant à la ruralité. Subséquemment, certains élus de municipalités d'importance en milieu rural ont eu l'idée de former un regroupement afin de se distinguer du lot de municipalités qui forment le territoire rural québécois.

\section{La problématique commune aux municipalités de centralité}

En 1998, un petit groupe d'élus municipaux se réunissaient afin de circonscrire la problématique commune aux municipalités de centralité. On peut dès lors parler du début du regroupement des municipalités que l'on disait à caractère de centralité. En décembre 2003, le regroupement est devenu le Caucus des municipalités de centralité qui s'est intégré dans la structure des caucus d'affinité de l'Union des municipalités du Québec (UMQ).

La problématique des municipalités de centralité est de ne pas être reconnues pour les fonctions de centralité qu'elles exercent. Réparties sur l'ensemble du territoire québécois, elles sont près d'une soixantaine. Ces agglomérations urbaines reçoivent quotidiennement la population des municipalités avoisinantes de la MRC pour y travailler, commercer, se récréer, étudier ou quérir des services de toutes natures. L'offre de services varie selon la taille de ces municipalités, mais cette donnée n'affecte en rien les principes intrinsèques d'influence et d'attraction de celles-ci.

\section{Les municipalités de centralité se retrouvent indistinctement dans les MRC dites à caractère rural et dans les MRC reconnues comme étant de caractère urbain et rural.}

Cette soixantaine de municipalités ne se perçoivent pas strictement que comme des centres de services. Elles sont aussi des pôles de développement qui doivent être reconnus comme tels et qui jouent un rôle moteur en rapport avec une collectivité élargie. En ce sens, nombre d'entre elles répondent parfaitement, sur une échelle réduite, à la définition statistique d'une agglomération. Mais au-delà des critères mathématiques, il y a fondamentalement toute une vie sociale et économique qui s'active autour d'elles et grâce à elles.

Comme mentionné plus haut, les enjeux de la réorganisation municipale ont forcé la caractérisation de la structure des municipalités locales sous deux types :

- les municipalités incluses dans une agglomération de recensement au sens statistique;

- les autres municipalités.

Les municipalités de centralité se retrouvent dans la seconde catégorie. À ce titre, elles sont confondues avec de petites entités sans aucun facteur d'influence, de développement ou d'attraction. Villages, paroisses et municipalités de centralité semblent présenter les mêmes caractéristiques pour le gouvernement. Au contraire, les municipalités de centralité se distinguent nettement par leur capacité de promouvoir et de développer l'essor économique régional. Elles sont le lieu de desserte des services gouvernementaux et paragouvernementaux. Elles concentrent la population de la MRC dans leurs industries et commerces pour qui elles sont le gagne-pain; elles abritent la population des aînés et des moins nantis dans leurs Offices municipaux d'habitation et leurs logements sociaux; elles divertissent la population régionale dans leurs équipements sportifs et culturels, etc.

Les municipalités de centralité se retrouvent indistinctement dans les MRC dites à caractère rural et dans les MRC reconnues comme étant de caractère urbain et rural, mais dans ce dernier cas, à l'extérieur des limites de l'agglomération de recensement. Autrement dit, elles sont toujours localisées dans le milieu rural de la MRC.

Pour exemple, mentionnons la ville de Maniwaki, située dans la MRC Vallée-de-la-Gatineau. Avec une population de 4097 habitants, elle concentre, parmi les 17 municipalités qui composent le territoire de la MRC, l'essentiel des services à la population. On y retrouve un Centre local de développement (CLD), un Centre local d'emploi (CLE), plusieurs établissements scolaires, des établissements de santé et de services sociaux, des équipements sportifs et culturels, un palais de justice et un aéroport régional. Cet ensemble d'équipements et d'établissements assure à la population de la MRC une accessibilité à une panoplie de services essentiels tant sociaux qu'économiques. 
Malgré un volume considérable de législations touchant l'organisation municipale, aucun texte n'a jusqu'ici proposé une définition distinctive de ces municipalités. Nulle part ne trouve-t-on une reconnaissance particulière du rôle et de la valeur du rôle de leadership et de direction rempli par celles-ci.

La politique de réorganisation municipale a plutôt souligné la nécessité de consolider indistinctement les centres de services en milieu rural. Les derniers développements législatifs n'ont fait que promouvoir le renforcement des pouvoirs et compétences des MRC. Cette initiative du parlement québécois n'aura, croiton, que pour seuls effets d'assurer une structure de soutien et de complémentarité aux collectivités locales de petite taille, dépossédées ou presque d'infrastructures pour servir le citoyen. À l'opposé, ces interventions parlementaires agiront négativement sur les municipalités de centralité en provoquant un affaiblissement de leurs pouvoirs décisionnels au profit du conseil de la MRC. Au surplus, ces réformes commanderont un support budgétaire accru de la part de la centralité au bénéfice de la MRC et des villages et paroisses.

Dans cette optique, le Caucus des municipalités de centralité souhaite obtenir la reconnaissance par statut officiel de l'existence de la centralité et de ses valeurs.

\section{La conceptualisation du caractère de centralité d'une municipalité}

Le concept de centralité ne pouvait demeurer dans un état primaire dans la mesure où une reconnaissance officielle était souhaitée. Il s'ensuit qu'une définition objective de la centralité doit permettre l'identifica- tion a priori des municipalités québécoises qui correspondent aux caractéristiques de centralité.

Ainsi, il a été convenu de retenir deux éléments fondamentaux de caractérisation de la centralité. Premièrement, les intéressés ont proposé et adopté une définition empirique qualitative de la centralité : «Carrefour des forces vives d'une communauté d'intérêts socio-économiques d'une région d'appartenance ou d'une partie de cette région qui, par ses capacités de polarisation et de rayonnement, concentre l'offre des services nécessaires et complémentaires à l'épanouissement d'une région ».

Deuxièmement, par une revue des critères statistiques, en l'occurrence le taux d'emplois tertiaires et de la présence d'équipements et d'institutions à vocation intermunicipale ou régionale, des critères spécifiques ont été choisis pour déterminer avec justesse et objectivité une définition empirique quantitative du caractère de centralité d'une municipalité :

- très forte centralité : 2000 emplois et plus du secteur tertiaire et 10 équipements ou institutions publics ou plus;

- forte centralité : de 1000 à moins de 2000 emplois du secteur tertiaire et 5 équipements ou institutions publics ou plus; ou de 500 à moins de $1000 \mathrm{em}-$ plois du secteur tertiaire et 10 équipements ou institutions publics ou plus.

En reprenant l'exemple de la Ville de Maniwaki, le tableau 1 illustre l'application des critères qualitatifs et quantitatifs pour la désignation de cette municipalité comme étant de centralité.

\section{Tableau 1 - Maniwaki}

\begin{tabular}{|c|c|c|c|c|c|}
\hline \multicolumn{6}{|c|}{ Équipements et institutions à vocation intermunicipale ou régionale ${ }^{2}$} \\
\hline $\begin{array}{l}\text { Développement } \\
\text { économique }\end{array}$ & $\begin{array}{l}\text { Établissements } \\
\text { scolaires }\end{array}$ & $\begin{array}{c}\text { Établissements de santé et } \\
\text { de services sociaux }\end{array}$ & $\begin{array}{l}\text { Culture } \\
\text { et loisirs }\end{array}$ & $\begin{array}{l}\text { Services } \\
\text { judiciaires }\end{array}$ & Divers \\
\hline 3 & 6 & 4 & 3 & 2 & 2 \\
\hline \multicolumn{6}{|c|}{ Données sociodémographiques ${ }^{3}$} \\
\hline \multicolumn{3}{|c|}{ Emplois totaux } & \multicolumn{2}{|c|}{ Emplois tertiaires } & \\
\hline \multicolumn{2}{|r|}{3265} & & \multicolumn{2}{|c|}{2625} & \\
\hline
\end{tabular}


Selon les données actuelles, qui seront mises à jour en fonction du recensement de 2001 de Statistique Canada et d'un nouveau recensement des équipements et institutions, 56 municipalités répondent aux critères de centralité (tableau 2).

\section{Tableau 2- Municipalités de centralité (par ordre alphabétique et par région administrative)}

$\begin{array}{cc}\text { Amqui (01) } & \text { Asbestos (05) } \\ \text { Mont-Joli (01) } & \text { Windsor (05) } \\ \text { Trois-Pistoles (01) } & \text { Richmond (05) } \\ \text { Saint-Pascal (01) } & \text { Coaticook (05) } \\ \text { La Pocatière (01) } & \text { Maniwaki (07) } \\ \text { Roberval (02) } & \text { Ville-Marie (08) } \\ \text { Saint-Félicien (02) } & \text { La Sarre (08) } \\ \text { La Malbaie (03) } & \text { Senneterre (08) } \\ \text { Baie-Saint-Paul (03) } & \text { Forestville (09) } \\ \text { Beaupré (03) } & \text { Port-Cartier (09) } \\ \text { Pont-Rouge (03) } & \text { Fermont (09) } \\ \text { Donnaconna (03) } & \text { Havre-Saint-Pierre (09) } \\ \text { Louiseville (04) } & \text { Chibougamau (10) } \\ \text { Lac-Mégantic (05) } & \text { Kuujjuak (10) }\end{array}$

\section{La caractérisation financière et fiscale des municipalités de centralité}

Avec l'adoption des critères précités de définition de la centralité, un pas était fait vers l'encadrement précis de la notion de centralité, mais la caractérisation restait vague. Tenter de définir la centralité en milieu rural revient en fait à s'interroger sur les caractéristiques d'un milieu urbain. En effet, les municipalités de centralité sont des municipalités urbaines au sein d'un milieu rural, et elles cherchent avant tout à se faire reconnaître comme telles, alors que les politiques gouvernementales les ont toujours assimilées à des municipalités rurales.

Par ailleurs, les municipalités québécoises tirent $75 \%$ de leurs revenus de leur richesse foncière, ces revenus servant à financer une panoplie de services locaux. Dès lors, la structure fiscale et financière d'une municipalité en dit long sur la nature et sur la répartition de sa richesse, ainsi que sur ses priorités ${ }^{4}$.
Îles-de-la-Madeleine (11)
Chandler (11)
Gaspé (11)

Ste-Anne-des-Monts (11)

New-Richmond (11)

Carleton-Saint-Omer (11)

Saint-Jean-Port-Joli (12)

Montmagny (12)

Sainte-Marie (12)

Beauceville (12)

St-Joseph-de-Beauce (12)

Lac-Etchemin (12)

Berthierville (14)

Rawdon (14)
Sainte-Adèle (15)

St-Sauveur-des-Monts (15)

Mont-Tremblant (15)

Mont-Laurier (15)

Farnham (16)

Waterloo (16)

Acton Vale (16)

Saint-Césaire (16)

Marieville (16)

Saint-Rémi (16)

Huntingdon (16)

Plessisville (17)

Nicolet (17)

Bécancour (17)
Ainsi, lorsqu'on se place du point de vue des structures politiques, l'une des avenues de différenciation entre l'urbain et le rural repose sur les caractéristiques fiscales et financières des municipalités qui composent ces milieux. C'est dans ce contexte que le Caucus des municipalités de centralité a décidé de se doter d'un diagnostic financier et fiscal afin de préciser sa définition et son rôle et ce, dans une perspective de développement économique en milieu rural. Une telle étude avait donc pour objectif d'habiller les municipalités d'une étiquette fiscale qui leur serait propre, ou du moins qui permettrait de les catégoriser et de les classifier.

Conformément à cet objectif, la question de recherche se résumait de la façon suivante : d'un point de vue fiscal et financier, qu'est-ce qui distingue les municipalités de centralité des autres municipalités en milieu rural ?

Pour réaliser cette recherche, l'UMQ s'est appuyée sur une compilation des rapports financiers annuels 
des 56 municipalités de centralité pour en comparer les résultats avec ceux de principalement deux autres catégories de municipalités ${ }^{5}$ : les municipalités rurales et les villes d'agglomération ${ }^{6}$.

Il ressort de cette recherche des résultats qui viennent confirmer et renforcer les constats empiriques préalables: les municipalités de centralité présentent des caractéristiques distinctives par rapport aux autres municipalités en milieu rural, ces caractéristiques reflétant leur rôle de centres de services et de pôles de développement en milieu rural ${ }^{7}$.

\section{La catégorisation fiscale}

La catégorisation fiscale est certainement la plus révélatrice des forces et faiblesses d'une municipalité en termes de richesse économique puisqu'elle en constitue la carte d'identité. Cette force de signification permet de définir à elle seule la centralité, alors que les autres éléments de catégorisation viennent renforcer cette définition. Deux indicateurs permettent d'établir une cartographie fiscale des municipalités de centralité : le premier concerne les bases de taxation, alors que le deuxième concerne les taux de taxation.

Premièrement, la richesse foncière révèle de grandes différences entre les municipalités de centralité et les autres municipalités en milieu rural. Les valeurs foncières des immeubles industriels et commerciaux en milieu rural sont fortement concentrées dans les municipalités de centralité, qui possèdent $40 \%$ des valeurs industrielles et commerciales situées en milieu rural, alors qu'elles ne comptent que $25 \%$ de la population en milieu rural. Aussi, les valeurs foncières des immeubles de la santé, des services sociaux et scolaires sont également fortement concentrées dans les municipalités de centralité, à hauteur de $54 \%$ pour la santé et les services sociaux, de $79 \%$ pour les cégeps et universités et de $43 \%$ pour le scolaire. Et surtout, il $\mathrm{y}$ a très peu de valeurs agricoles dans les municipalités de centralité, qui ne possèdent que $7 \%$ de l'ensemble des valeurs agricoles en milieu rural. Pour continuer avec l'exemple de Maniwaki, cette municipalité témoigne bien de ces résultats puisque les valeurs industrielles et commerciales comptent pour $23 \%$ de l'ensemble des valeurs foncières inscrites au rôle, alors qu'elles ne comptent que pour $9 \%$ de l'ensemble des valeurs foncières des autres municipalités de la MRC Vallée-de-la-Gatineau. De même, les va- leurs agricoles comptent pour moins de $1 \%$ à Maniwaki, contre plus de $4 \%$ ailleurs dans la MRC. Ces résultats dessinent un portrait sans ambiguïté des municipalités de centralité : un milieu qui concentre les emplois et les services publics et qui est entouré d'un territoire majoritairement agricole et résidentiel.

\section{Deux indicateurs permettent d'établir une cartographie fiscale des municipalités de centralité.}

Deuxièmement, le fardeau fiscal imposé aux contribuables locaux est beaucoup plus élevé dans les municipalités de centralité. En effet, l'effort fiscal fourni par les municipalités de centralité, mesuré par le taux global de taxation uniformisé ${ }^{8}$, s'établit à $1,84 \$$ par 100 \$ d'évaluation, en comparaison de 1,34 \$ pour les municipalités rurales. Encore une fois, Maniwaki est un bon exemple de cette réalité, avec un effort fiscal de $3,15 \$$ en comparaison de $1,28 \$$ par $100 \$$ d'évaluation pour les autres municipalités de la MRC. Cette différence s'explique facilement. Les municipalités de centralité doivent offrir une large gamme de services, ce qui explique des taux de taxation élevés. De fait, les habitants ont tendance à s'installer dans les municipalités rurales avoisinantes, où les taux de taxation sont plus faibles en raison du niveau inférieur de services. Mais ces mêmes habitants viennent utiliser les services des municipalités de centralité, ce qui crée une pression vers l'augmentation du taux de taxation. Cet écart rappelle celui qui existait, avant la réorganisation municipale, entre villes-centres et banlieues.

\section{La catégorisation financière}

Partant d'un objectif d'analyse structurelle, notre recherche s'est d'abord tournée vers l'aspect financement des investissements du budget des municipalités pour des conclusions sur la catégorisation financière des municipalités de centralité.

Le niveau d'endettement d'une municipalité donnée constitue un indicateur de ses marges de manœuvre financières actuelles et futures. C'est pourquoi nous l'avons analysé à partir de ratios communément utilisés, soit l'endettement par rapport à la richesse foncière et le poids du service de la dette dans l'ensemble des dépenses. Continuons avec Maniwaki, dont la dette per capita était de $510 \$$ en 2001 , alors qu'elle 
était de $220 \$$ dans les autres municipalités de la MRC. Les résultats de ces ratios montrent que les municipalités de centralité sont globalement plus endettées que les autres municipalités en milieu rural, ce qui est conforme à la définition de la centralité, puisque ces villes possèdent plus d'infrastructures et d'équipements qui desservent aussi la population des autres municipalités. En effet, plus la ville est urbanisée, plus son niveau d'investissement et son niveau d'endettement sont élevés.

Quant aux subventions, il s'agit de transferts du gouvernement conditionnels à la réalisation d'investissements très spécifiques visés par un programme particulier. En 2001, les municipalités de centralité ont reçu seulement $15 \%$ de l'ensemble des transferts conditionnels versés aux municipalités en milieu rural, ce qui représente $84 \$$ par habitant, contre $143 \$$ par habitant pour l'ensemble des municipalités en milieu rural. À Maniwaki, les transferts conditionnels représentent $46 \$$ par habitant. Cette comparaison nous permet de tirer la conclusion que les municipalités de centralité sont moins dépendantes des transferts gouvernementaux pour la réalisation d'investissements que leurs consœurs rurales.

\section{Plus la ville est urbanisée, plus son niveau d'investissement et son niveau d'endettement sont élevés.}

Ensuite, la recherche s'est penchée sur la structure de dépenses des municipalités de centralité. L'objectif de cet article étant de camper la définition des municipalités de centralité, nous épargnerons aux lecteurs une présentation détaillée de la structure de dépenses et nous en rapportons ici un exemple qui nous semble révélateur : les municipalités de centralité consacrent près de $15 \%$ de leur budget aux dépenses de loisirs et culture, contre seulement $9 \%$ chez les municipalités rurales. La municipalité de Maniwaki consacre près de $16 \%$ de son budget aux dépenses de loisirs et de culture. Ces dernières comprennent les dépenses des centres communautaires, des patinoires et piscines, des parcs et des bibliothèques. Ces dépenses représentent 139 \$ par habitant dans les municipalités de centralité. La présence d'équipements de loisirs et de culture est l'une des caractéristiques des municipalités de centralité par rapport aux municipalités voisines, il n'est donc pas surprenant que les dépenses de fonc- tionnement relatives à ces équipements soient aussi plus élevées. Rappelons que plusieurs de ces équipements ont une vocation supralocale. L'analyse sommaire des dépenses de fonctionnement par type de service au citoyen des municipalités de centralité nous montre que leur profil est très différent de celui des municipalités rurales. Une analyse plus fine sera peutêtre possible une fois que les indicateurs de gestion municipaux auront été mis en application, ces indicateurs permettant de comparer les services municipaux entre plusieurs municipalités en tenant compte de facteurs d'influence majeurs comme la densité de population.

\section{Synthèse du diagnostic financier et fiscal}

Les municipalités de centralité possèdent une structure financière et fiscale très différente de celle des municipalités rurales, structure qui se rapproche de celle des villes d'agglomération. En effet, chacun des résultats présentés ci-dessus a été comparé à ceux des villes d'agglomération, cette comparaison ayant révélé une très grande similarité entre centralité et agglomération, d'un point de vue fiscal et financier. Tout comme les villes d'agglomération, les municipalités de centralité possèdent donc une structure financière et fiscale qui témoigne de leur rôle de centres de services et de pôles d'influence et de développement. Les différences entre les deux catégories sont plus de l'ordre de l'échelle de population, et certainement du périmètre de rayonnement, que l'on peut présumer plus grands pour les villes d'agglomération.

\section{La dynamique des relations entre municipalités et MRC en milieu rural}

Dans un contexte de transferts potentiels de responsabilités des municipalités vers les MRC, l'objectif de cette étude était aussi d'évaluer l'impact de ces transferts pour les municipalités de centralité. Les derniers développements législatifs n'ont fait que promouvoir le renforcement des pouvoirs et des compétences des MRC, alors que les municipalités de centralité croient que le renforcement des compétences des MRC au détriment des municipalités provoquera un affaiblissement de leur pouvoir décisionnel, tout en leur demandant un soutien budgétaire accru au profit de la MRC et des municipalités rurales les composant. Ainsi, plusieurs compétences ont été transférées des munici- 
palités aux MRC de façon obligatoire et d'autres de façon facultative à la suite de l'adoption du projet de loi 29 en 2001, de l'adoption de la politique de la ruralité en 2002, puis de l'adoption du projet de loi 77 en 2002.

Les transferts de compétences des municipalités vers les MRC que nous avons cherché à analyser sont très récents, et il était encore trop tôt pour en mesurer véritablement les impacts. Par contre, afin de se faire une idée de l'ampleur possible de ces impacts, nous avons tenté de chiffrer combien représentaient les responsabilités transférées dans les budgets des municipalités de centralité en 2001.

Pour les municipalités de centralité, les dépenses liées aux responsabilités en cours de transfert aux MRC sont de plus de 66 millions de dollars en 2001, ce qui représente près de $20 \%$ de leurs dépenses d'opération, sans oublier les parcs régionaux et autres équipements supralocaux, qui ne sont pas inclus dans ces $20 \%$ (tableau 3).

Tableau 3 - Transferts de compétences des municipalités vers les MRC

\begin{tabular}{|c|c|c|}
\hline $\begin{array}{c}\text { Responsabilité transférée } \\
\text { à la MRC }\end{array}$ & $\begin{array}{l}\text { Montants inscrits au rapport } \\
\text { financier 2001- ensemble des } \\
\text { municipalités du Québec }\end{array}$ & $\begin{array}{l}\text { Dont municipalités de centralité } \\
\text { (évaluation UMQ) }\end{array}$ \\
\hline Évaluation foncière & $85,6 \mathrm{M} \$$ & $4,4 \mathrm{M} \$$ \\
\hline Transport collectif & $506,4 \mathrm{M} \$$ & $5,0 \mathrm{M} \$$ \\
\hline Gestion des cours d'eau & $1,9 \mathrm{M} \$$ & $0,131 \mathrm{M} \$$ \\
\hline Gestion des matières résiduelles & $394,4 \mathrm{M} \$$ & $23,7 \mathrm{M} \$$ \\
\hline Gestion de la voirie & $492,0 \mathrm{M} \$$ & $31,2 \mathrm{M} \$$ \\
\hline Logement social & $19,0 \mathrm{M} \$$ & $1,7 \mathrm{M} \$$ \\
\hline Parcs régionaux & $? ?$ & \\
\hline $\begin{array}{l}\text { Équipements, infrastructures et } \\
\text { activités à caractère supralocal }\end{array}$ & $? ?$ & \\
\hline TOTAL & $1499,3 \mathrm{MS}$ & $66,1 \mathrm{MS}$ \\
\hline
\end{tabular}

Ces résultats nous amènent à conclure que non seulement les municipalités de centralité pourraient perdre $20 \%$ de leur pouvoir décisionnel, dans des domaines très divers des services à la population, mais en plus que ce transfert risque de créer des dédoublements. Par exemple, le personnel affecté à ces services ne pourra pas être complètement transféré à la MRC. Or, rappelons-nous que les dépenses de personnel représentent $37 \%$ des budgets municipaux. En ce qui concerne Maniwaki, les dépenses d'opération liées aux responsabilités à transférer aux MRC représentent $27 \%$ de son budget.

Les transferts de compétences des municipalités vers les MRC risquent par conséquent de fragiliser les finances des villes qui possèdent actuellement les services compétents pour assumer ces responsabilités, sans toutefois posséder une masse critique d'em- ployés, et ce sont les municipalités de centralité qui correspondent à cette situation.

Ces hypothèses sont renforcées par le fait que les municipalités rurales sont en général dépendantes des services offerts par les municipalités de centralité, ces dernières percevant $40 \%$ des revenus de services rendus via des ententes intermunicipales en milieu rural, lesquelles concernent principalement les services en hygiène du milieu (eau potable, eaux usées), incluant la gestion des matières résiduelles et les services de sécurité incendie. De nombreuses ententes sont également conclues en matière de loisirs, de culture et de patrimoine, venant confirmer le rôle de centre de services des municipalités de centralité. Notre municipalité témoin, Maniwaki, répond à cette définition puisqu'elle dispense des services dans le cadre de neuf ententes pour des services très variés allant de la 
gestion des matières résiduelles aux services de loisirs.

Plus globalement, ces transferts de compétences vers la MRC posent la question de la légitimité et du rôle de ce palier d'administration locale.

\section{Conclusion}

La recherche de reconnaissance de la part des municipalités de centralité s'inscrit dans une volonté de renforcer le développement des milieux ruraux dans lesquels elles se situent. Dans cet esprit, les recherches effectuées pour ce groupe de municipalités tentent de démontrer qu'il est possible et souhaitable de travailler sur la définition de la centralité sans être en contradiction avec la MRC, et de travailler en collaboration avec la ruralité. Ces recherches visent en effet à présenter les avantages de la centralité pour la région toute entière, plutôt que les méfaits du renforcement de la MRC.

Finalement, nous croyons qu'il est souhaitable de substituer la politique de renforcement des MRC par une politique de renforcement régional.

Ainsi, la reconnaissance des municipalités de centralité devra être assurée par le gouvernement du Québec et ce, pour éviter que ces municipalités ne connaissent de graves difficultés financières et économiques, mais surtout afin d'assurer le renforcement régional des secteurs ruraux, dont la vitalité dépend beaucoup de l'existence et de la vitalité de pôles de services et de développement à proximité.

Les données financières et fiscales sont en effet nombreuses pour venir réaffirmer que les municipalités de centralité représentent une catégorie particulière, différente des municipalités rurales. Ce sont des municipalités avec un caractère plus urbain de centre de services et de pôle d'influence et de développement qui seraient plus comparables à ce que sont les villes d'agglomération. Les données financières et fiscales montrent également que le transfert de compétences aux MRC affaiblira sans aucun doute les municipali- tés de centralité, tant en termes de contrôle sur leur budget qu'en termes de coût d'un tel transfert.

L'affaiblissement des municipalités de centralité serait d'autant plus dramatique pour le milieu rural s'il entraînait en même temps une série d'obstacles et de freins à son développement et au développement régional. Pour cette raison, nous avons complété la recherche par une analyse socio-économique s'appuyant sur des données autres que financières. Les résultats de cette recherche complémentaire seront présentés dans le prochain numéro de la revue.

\section{Notes et références}

1 Peggy Bachman est conseillère en fiscalité à la direction des Politiques de l'UMQ et doctorante en administration à l'Université de Sherbrooke. Jean Langevin est conseiller aux politiques à la direction des Politiques de l'UMQ.

2 Selon des données recueillies principalement parmi les recensements officiels du gouvernement du Québec.

3 Selon les données du recensement de 1996 de Statistique Canada.

4 Voir sur ce point : Collin, J-P et P.J. Hamel (1993). « Les contraintes structurelles des finances publiques locales : les budgets municipaux dans la région de Montréal en 1991 ", Recherches sociographiques, vol. XXXIV, $\mathrm{n}^{\circ} 3$, p. 439467.

5 Cette comparaison a été possible grâce aux résultats de l'étude conjointe UMQ - Conference Board du Canada (2003). La situation fiscale des municipalités québécoises. Rapport complet.

6 Il existe 26 villes d'agglomération au Québec qui correspondent à la définition des agglomérations de recensement (AR) de Statistique Canada.

7 Les résultats complets de cette recherche sont disponibles à www.umq.qc.ca.

8 Ce calcul consiste à diviser les revenus d'imposition (exclusion faite de la taxe sur les immeubles non résidentiels, de la surtaxe sur les immeubles non résidentiels et de la taxe d'affaires) par l'évaluation uniformisée des immeubles imposables. On obtient alors le taux théorique qu'il serait nécessaire d'imposer si l'ensemble des revenus de taxation de la municipalité provenait d'une taxe générale imposée sur la valeur de tous les immeubles imposables. 